\title{
A Prerequisite for Life
}

\author{
Søren Toxvaerd \\ Department of Science and Environment, Roskilde University, Postbox 260, Roskilde DK-4000, Denmark
}

\section{A R T I C L E I N F O}

\section{Article history:}

Received 17 December 2018

Revised 28 March 2019

Accepted 2 May 2019

Available online 3 May 2019

Keywords:

Abiogenesis

Homochirality

Prebiotic environment

\begin{abstract}
A B S T R A C T
The complex physicochemical structures and chemical reactions in living organism have some common features: (1) The life processes take place in the cytosol in the cells, which, from a physicochemical point of view is an emulsion of biomolecules in a dilute aqueous suspension. (2) All living systems are homochiral with respect to the units of amino acids and carbohydrates, but (some) proteins are chiral unstable in the cytosol. (3) And living organism are mortal. These three common features together give a prerequisite for the prebiotic self-assembly at the start of the Abiogenesis. Here we argue, that it all together indicates, that the prebiotic self-assembly of structures and reactions took place in a more saline environment, whereby the homochirality of proteins not only could be obtained, but also preserved. A more saline environment for the prebiotic self-assembly of organic molecules and establishment of biochemical reactions could have been the hydrothermal vents.
\end{abstract}

(c) 2019 Published by Elsevier Ltd.

\section{Introduction}

Numerous articles deal with the environmental and physicochemical conditions for -, and with the chemical reactions at the establishment of life on planet Earth, or somewhere else in the universe (Bada, 2004; Lane et al., 2010; Huber et al., 2012; Hud et al., 2013; Pascal et al., 2013; Stüeken et al., 2013; Sousa et al., 2013; Sutherland, 2017; Spitzer, 2017; Walker, 2017; Branscomb and Russell, 2018). Many of the suggestions are inspired by Darwin, and link these conditions and reactions with the chemical composition and reactions in LUCA, our last universal common ancestor (Velasco, 2018; Higashi et al., 2018). It is, however, not clear whether LUCA is a simple Archaea or Bacteria, or whether it is a more primitive organism, a Progenote Woese and E. (1977); Woese (1987); Giulio (2011); Weiss et al. (2016); Giulio (2018). For a recent review see Weiss et al. (2018). But although LUCA, superficially looks rather simple, it is in fact very complicated and highly structured (Weiss et al., 2016; 2018), and with all the fundamental preconditions for our life. LUCA has a genetics and a metabolism controlled by enzymes in a cell with ion channels and active transport.

Our first ancestor is at least 3.5 billion years (Ga) old Djokic et al. (2017); Schopf et al. (2018), and there is indirect signs of life even before 3.5 Ga Tashiro et al. (2017); Retallack and Noffke (2019). The earth is $\approx 4.6 \mathrm{Ga}$ old and there is good evidence for, that the oceans were established shortly after, maybe after few hundred million years (Harrison, 2009; Sleep, 2010). The prebiotic

\footnotetext{
E-mail address: st@ruc.dk
}

time defined as the time interval, from where the physicochemical conditions allowed for an inorganic and organic self-assembly of bio-materials to the time, where LUCA appeared, is most likely millions of years. This article deals with the start of the Abiogenesis: the spontaneous chemical self-assembly of the organic building units and the establishment of reactions in a prebiotic environment.

There are many universal properties of all the living species, which might guide us in establishing a prerequisite for life. On one hand living species are very complex, even in the details. Take for instance the metabolism, the genetics or our immune system, or... But on the other hand they have some common features. All living species are from a thermodynamic point of view open "driven" system far from equilibrium. The biochemical reactions in the cells are in a diluted aqueous (cytosol) solution (1), the units of the peptides and carbohydrates are all homochiral units (2), and living species are mortal (3). There are many other common features of biosystems. Beside of the metabolism and the genetics with the DNA and RNA, also e.g. the selfreplication and the cells with membranes. And with respect to mortality there exists some counterexamples of living organisms with a remarkable resistance to aging. For instance some primitive organisms, Hydra vulgaris, show no sign of mortality for a period of four years (Martínez, 1998), and some jellyfish perform life cycle reversal (He et al., 2015). Another example is the stability of the enzyme telomerase and the telomere. Together they are responsible for the unlimited proliferation of almost all cancer cells (Cong et al., 2002).

With respect to the necessity of homochirality of the units of amino acids and carbohydrates, it is in general not debated in arti- 
cles, which deal with the prerequisites for life. But here we argue, that the three common properties: the composition of the cytosol, the instability of homochirality in enzymes and mortal instability of living systems, are connected, and that they together also is a key to determine a prerequisite for our life: prebiotic self-assembly of homochiral enzymes in a saline aqueous environment.

\section{The aqueous cytosol solution in the cells, homochirality and life}

All the cells in living organisms are soft condensed matter, limited by cell membranes and the cells contain an aqueous (cytosol) suspension of organic molecules. The cytosol can be characterized as a diluted aqueous solution of cat- and anions at a total concentration (exclusive amino acids) of $\approx 0.15 \mathrm{M}$ of $\left[\mathrm{Na}^{+}\right] \approx$ $0.01 \mathrm{M},\left[\mathrm{K}^{+}\right] \approx 0.10 \mathrm{M},\left[\mathrm{HCO}_{3}^{-}\right] \approx 0.008 \mathrm{M}$, and other ions, $\mathrm{Cl}^{-}$, $\mathrm{Ca}^{++}, \mathrm{Mg}^{++}$, , with smaller concentrations. Despite the small ionic concentrations in the cell interior, the cytostol with a complex cytoplasm with water networks and hyperstructures (Shepherd, 2006; Norris et al., 2007), departs physicochemical from the condition of an ideal aqueous solution Luby-Phelps (2000).

LUCA is at least 3.5 billion years (Ga) old, and it is therefore natural to describe how the earth was, and how water appeared, at that time. The earth is $\approx 4.6 \mathrm{Ga}$ old and the Hadean eon is the period from $4.6 \mathrm{Ga}$ to $4.0 \mathrm{Ga}$, followed by the Achaean eon from $4.0 \mathrm{Ga}$ to $2.5 \mathrm{Ga}$. There is evidence for, that the ocean(s) were established relatively shortly after $4.6 \mathrm{Ga}$, maybe after one hundred million years Harrison (2009); Sleep (2010). The estimates of the physicochemical state of the water in the oceans after the establishment varies from prediction of an extreme salty and acidic Hadean ocean (Maruyama et al., 2013; Aoki et al., 2018) to an Archean ocean, with a salt content as in the present oceans (Marty et al., 2018). Today the oceans contain a sodium concentration of $\left[\mathrm{Na}^{+}\right] \approx 0.47 \mathrm{M}$ and $\left[\mathrm{K}^{+}\right] \approx 10^{-2} \mathrm{M}$. The difference between the concentrations of sodium and potassium outside and inside the cell is vital and maintained by the sodium pump.

Living systems consist of peptides with units of L-amino acids and D-carbohydrates. The amino acids have, however, an active isomerization kinetics and will racemize in an aqueous solution (Bada, 1972). The chirality of a peptide in a cell is also unstable (Fujii et al., 2018). But from computer simulations and thermodynamic investigations of aqueous suspensions of peptide-like molecules at different water activities one finds, that although peptides lose their homochirality at high water activity, they are stable and maintain homochirality at a lower water activity (saline solutions) (Toxvaerd, 2017), in accordance with the observation of the stability of homochirality in a peptide. The physicochemical explanation for this behavior is, that the compact $\alpha$-helix structure, predicted by Pauling et al. (1951); Pauling and Corey (1951), ensures a sufficient chiral discrimination for obtaining and maintaining homochirality, but provided that the water activity is sufficiently low (Toxvaerd, 2018). According to Pauling, the $\alpha$-helix structure is ensured by weak hydrogen-like bonds between units in the helix. But these bonds are in competition with hydrogen bonds to the attached water molecules at the protein, which destabilizes the $\alpha$-helix structure at high water activity (i.e. concentration).

It is, however, only the stability of homochirality in the proteins, which depends on the salinity of the aqueous cytosol suspension, because the homochirality of carbohydrates is obtained and ensured in a different way, by stereo specific enzymes in the metabolism Toxvaerd (2018). All polymer units in carbohydrates have a D-configuration at carbon atom No. 5 for hexose-units and No. 4 for pentose-units. The carbohydrate polymers in biosystems are synthesized from Glyceraldehyde-3-phosphate, which has a very active isomerization kinetics. The isomerization kinetics for Glyceraldehyde-3-phosphate is catalyzed by the extreme effective enzyme, Triose Phosphate Isomerase, and it looks like a paradox, that the bio-carbohydrate wold is $100 \%$ homochiral, when the key-molecule in the polymerization is chiral unstable. But the explanation of this paradox is, that once the polymerization with Glyceraldehyde-3-phosphate starts by creation of a hexose or a pentose (Ribose), the chiral center in Glyseraldehyde-3-phosphate is preserved by stable covalent bonds (Toxvaerd, 2014), and the total dominance of the D-configurations in bio-carbohydrates is obtained and maintained by stereo specific enzymes (Toxvaerd, 2018).

That proteins and enzymes are homochiral stable at a low water activity, is a daily life's observation. One can conserve meat in a salty- or sugar solutions, whereby the bacteria are dehydrated and inactive. But their life is only set on "stand-by" in the salty solution, and some bacteria can even survive in a dehydrated state under extreme conditions of pressure and temperature for a very long time (Nicholson et al., 2000). It is therefore natural to conclude, that the prebiotic self-assembly of peptides from units of amino acids took place in an environment with higher salinity and lower water activity than in the cytosol, whereby one not only could obtain, but also maintain homochirality by the peptide synthesis (Toxvaerd, 2017).

\section{Darwin's warm little pond}

There has been many proposals to the geographic location(s), where life occurred. The Hadean ocean(s), tidal pools, icy environments, mineral surfaces, alkaline hydrothermal vents, to mention some proposals, which also includes extraterrestrial places. This article focus on the necessity of a rather salty solution with a relative high concentration of the building blocks of amino acids in order to ensure a sufficient strength of chiral discrimination at the peptide polymerization. It has always been a puzzle, from where the amino acids came, but in fact this question is irrelevant for the start of the Abiogenesis. The problem is not from where they came, or whether or not the amino acids were in a racemic or homochiral composition. The amino acids have an active isomerization kinetics, and an aqueous solution of amino acids will racemize over time Bada (1972). The problem is how to maintain a sufficient high concentration of amino acids for the polymerization, and to how ensure a homochiral stable form for a very long time. So long to that the other self-assembly of higher order structures and consecutive reactions in bio systems could be established. The necessity of a high concentration of amino acids exclude the bulk ocean(s) and points to places with "confine geometry". Because, if the prebiodic polymerization of peptides had occurred in a well stirred Hadean ocean, it would require an enormous amount of amino acids.

In a letter to a close friend Darwin proposed, that life were started “..in some warm little pond." Peretó et al. (2009), and since then there has been many suggestions to where in the aqueous environment the life processes started. The Darwinism is often presented as a continuous coherent evolution from simple inorganic processes toward the living systems. This outlook of the emergence of living systems as prebiotic " chemistry in a bag" has been criticized Branscomb and Russell (2018). Branscomb and Russell noticed, that all living systems exist in a self-generate physical state, that is extremely far from thermodynamic equilibrium i.e., equivalently, of extremely low entropy and thus of correspondingly low probability. They conclude, that a simple mass-action chemistry cannot explain the self-generated and far-from -equilibrium myriad of disequilibrium in a living organism.

The living organism is in a " far from equilibrium" state with gradients in the electrical potential, and protonic and ionic concentrations. These facts excludes most of the proposed locations for the prebiotic self-assembly of the building blocks. The most favorable place, however, is the submarine 
alkaline hydrothermal vents, where the confined environment with protonic, ionic and concentration gradients fulfil the requirements for non-equilibrium self-assembly of higher order organic structures Sousa et al. (2013); Branscomb and Russell (2018); Russell et al. (1994); Sleep et al. (2011). In this context it is interesting to notice, that the earliest sign of life is located to be in fluvial hot spring deposits Djokic et al. (2017).

It is natural to connect the evolution of bio systems with the geological evolution. The geology of the planet Earth has changed significantly over time, and the physicochemical environment, where the prebiotic self-assembly took place, must also have changed during the prebiotic time. This fact might explain how the strong non-equilibrium chemistry with differences in ion concentrations between the cytosol and the aqueous environment have been established. Could it not have happened, that e.g. emulsion of peptides were synthesized as "chemistry in a bag" in the vents or in the confine geometry below the vents, and then were exposed to drastic changes in concentrations caused be changes in the vents? LUCA is a space shuttle, not a shuttle in an empty space, but " chemistry in a bag" in a hostile salty sea. We are used to consider the sodium pump as a physicochemical mechanism to ensure a potential difference at the membrane and a gradient in sodium and potassium. But the ion channels also ensure a low salinity in the cells, which is a necessity for our life processes.

\section{Discussion}

The Abiogenesis, or the origin of life, is probably not a result of a series of single events, but rather the result of a gradual process with increasing complexity of molecules and chemical reactions, and the prebiotic synthesis might not have left any trace of the establishment of structures and reactions at the beginning of the evolution Hud et al. (2013); Fialho et al. (2018); Raiser (2018). But the evolution have lead to different forms of the most simple living systems. There exists two forms of simple procariotes: bacteria and the archaea, who mainly differ with respect to the constitutions of their membranes. Their metabolism, genetics and enzyme systems are qualitative the same, and they are both homochiral with respect to their peptide and carbohydrate units and with an interior of the cells, which is a diluted aqueous suspension. These facts seems to indicate, that the prebiotic biosynthesis, the metabolism and a genetics were established to some extent, before the life were established. E.g. the central enzyme in Glycolysis/gluconeogenesis, Triose Phosphate Isomerase may have been present in the proteome de Farias et al. (2016). And the very fist step in polymerization of carbohydrates in the metabolism, the synthesis of D-fructose-1,6-bisphosphate from dihydroxyacetone phosphate and D-Glyceraldehyde-3-phosphate is controlled by a stereo specific enzyme, Aldolase Munegumi (2015). An enzyme which also can be identified as an ancestral enzyme Say and Fuchs (2010).

The missing link between a saline prebiotic environment and LUCA is the advent of life: a cell with a membrane, metabolism and genetics. A cell, who although it was unstable, was self generating, whereby a wave of life were started.

One might object, that the hypothesis about the emergence of homochirality in a prebiotic saline aqueous environment is speculation, without a possibility of verification. This is, however, not correct. Some part of the hypothesis can be experimentally verified. It is straight forward possible to test for homochiral stability of enzymes with respect to the activity of water. The prediction is that the homochiral stability of enzymes depends on the degree of salinity of the aqueous suspension. One shall expect, that an enzyme in pure water is more chiral unstable than in an in vitro cytosol solution, and that it is stable at a higher ionic concentration than in the cytosol.
The hypothesis is also, that the stability of homochirality in proteins is a necessity for obtaining and maintaining the homochirality of the carbohydrates Toxvaerd (2018). It might also be possible to verify this part of the hypothesis: That the metabolism only acts with stereo specific enzymes, whereby the homochirality of carbohydrates is ensured by the kinetics in the metabolism Toxvaerd (2018). The prediction is that also Ribose's metabolism is controlled by stereo specific enzymes, which ensures a D-Ribose wold, a necessity for RNA and DNA. And that these enzymes are ancestral and present in the Progenote and in LUCA. If so, it establish an order in the evolution Toxvaerd (2018), and these behaviors together links the physicochemical state of the cytosol in the living cell together with the instability of homochirality and the dead of a living organism in the global wave of life.

And it gives an ideas of, where in the aqueous environment and how the prebiotic self-assembly took place.

\section{Acknowledgment}

Jeppe C Dyre is gratefully acknowledged. This work was supported by the VILLUM Foundation's Matter project, grant No. 16515.

\section{References}

Aoki, S., Kabashima, C., Kato, Y., Hirata, T., Komiya, T., 2018. Influence of contamination on banded iron formations in the isua supracrustal belt, west greenland: reevaluation of the eoarchean seawater compositions. Geosci. Front. 9, 1049-1072.

Bada, J.L., 1972. 1972. Kinetics of racemization of amino acids as a function of ph. J. Am. Chem. Soc. 94, 1371-1373.

Bada, J.L., 2004. How life began on earth: a status report. Earth Planet. Sci. Lett. 226 $1-15$.

Branscomb, E., Russell, M.J., 2018. Frankenstein or a submarine alkaline vent: who is responsible for abiogenesis? BioEssays 40, 1700179-1700182.

Cong, Y.-S., Wright, W.E., Shay, J.W., 2002. Human telomerase and its regulation. Microbiol. Mol. Biol. Rev. 66, 407-425.

Djokic, T., Van Kranendonk, M.J., Campbell, K.A., Walter, M.R., Ward, C.R., 2017. Earlist signs of life on land preserved in ca. 3.5 ga hot spring deposits. Nature Commun. 8, 15263.

de Farias, S.T., Rêgo, T.G., José, M., 2016. A proposal of the proteome before the last universal common ancestor (LUCA). Intern. J. Astrobiol. 15, 27-31.

Fialho, D.M., Clarke, K.C., Moore, M.K., Schuster, G.B., Krishnamurthy, R., Hud, N.V., 2018. Glycosylation of a model proto-RNA nucleobase with non-ribose sugars: implications for the prebiotic synthesis of nucleotides. Org. Biomol. Chem. 16, 1263-1271.

Fujii, N., Takata, T., Fujii, N., Aki, K., Sakaue, H., 2018. D-Amino acids in protein the mirror of life as a molecular index of aging. BBA-Proteins Proteomics 1866 840-847. 1,6-bisphosphate aldolase/phosphatase may be an ancestral gluconeogenic enzyme. Nature 464, 1077-1081

Giulio, M.D., 2011. The last universal common ancestor (LUCA) and the ancestors of archaea and bacteria were progenotes. J. Mol. Evol. 72, 119-126.

Giulio, M.D., 2018. On earth, there would be a number of fundamental kinds of primary cells - cellular domains - greater than or equal to four. J. Theor. Biol. 443, 10-17.

Harrison, T.M., 2009. The hadean crust: evidence from $>4$ ga zircons. Annu. Rev. Earth Planet. Sci. 37, 479-505

He, J.H., Zheng, L., Zhang, W., Lin, Y., 2015. Life cycle reversal in Aurelia sp. 1(cnidaria scyphozoa). PLoS ONE 10 (12), e0145314.

Higashi, K., Kawai, Y., Baba, T., Kurokawa, K., Oshima, T., 2018. Essential cellular modules for the proliferation of the primitive cell. Geosci. Front. 9, 1155-1161.

Huber, C., Kraus, F., Hanzlik, M., Eisenreich, W., Wächtershäuser, G., 2012. Elements of Metabolic Evolution. Chem. Eur. J. 18, 2063-2080.

Hud, N.V., Cafferty, B.J., Krishnamurthy, R., Williams, L.D., 2013. The origin of RNA and "my grandfather's axe". Chem. Biol. 20, 466-474.

Lane, N., Allen, J.F., Martin, W., 2010. How did LUCA make a living? Chemiosmosis in the origin of life. BioEssays 32, 271-280.

Luby-Phelps, K., 2000. Cytoarchitecture and physical properties of cytoplasm: volume, viscosity, diffusion, intracellular surface area. . Int. Rev. Cytol. 192, 189-221.

Martínez, D.E., 1998. Mortality patterns suggest lack of senescence in hydra. Exp. Gerontol. 33, 217-225.

Marty, B., Avice, G., Bekaert, D.V., Broadley, M.W., 2018. Salinity of the achaean oceans from analysis of fluid inclusions in quartz. C. R. Geo. Sci. 350, 154-163.

Maruyama, S., Ikoma, M., Genda, H., Hirose, K., Yokoyama, T., Santosh, M., 2013. The naked planet earth: most essential pre-requisite for the origin and evolution of life. Geosci. Front. 4, 141-165.

Munegumi, T., 2015. Aldolase as a chirality intersection of l-amino acids and d-sugars. Orig. Life. Evol. Biosph. 45, 173-182. 
Nicholson, W.L., Munakata, N., Horneck, G., Melosh, H.J., Setlow, P., 2000. Resistance of Bacillus endospores to extreme terrestrial and extraterrestrial environments. Microbiol. Mol. Biol. Rev. 64, 548-572.

Norris, V., den Blaauwen, T., Cabin-Flaman, A., Doi, R.H., Harshey, R., Janniere, L., Jimenez-Sanchez, A., Jin, D.J., Levin, P.A., Mileykovskaya, E., Minsky, A., Saier Jr., M., Skarstad, K., 2007. Functional taxonomy of bacterial hyperstructures. Microbiol. Mol. Biol. Rev. 71, 230-253.

Pascal, R., Pross, A., Sutherland, J.D., 2013. Towards an evolutionary theory of the origin of life based on kinetics and thermodynamics. Open Biol 3, 130156.

Pauling, L., Corey, R.B., 1951. The pleated sheet, a new layer configuration of polypeptide chains. Proc. Natl. Acad. Sci 37, 251-256.

Pauling, L., Corey, R.B., Branson, H.R., 1951. The structure of proteins: two hydrogen-bonded helical configurations of the polypeptide chain. Proc. Natl. Acad. Sci. 37, 205-211.

Peretó, J., Bada, J.L., Lazcano, A., 2009. Charles darwin and the origin of life. Orig. Life. Evol. Biosph. 39, 395-406.

Raiser, M., 2018. An appeal to magic? the discovery of a non-enzymatic metabolism and its role in the origins of life. Biochemical J. 475, 2577-2592.

Retallack, G.J., Noffke, N., 2019. Are there ancient soils in the 3.7 ga isua greenstone belt, greenland? Palaeogeogr., Palaeoclimatol., Palaeoecol. 514, 18-30.

Russell, M.J., Daniel, R.M., Hall, A.J., Sherringham, J.A., 1994. A hydrothermally precipitated catalytic iron sulphide membrane as a first step toward life. J. Mol. Evol. 39, 231-243.

Say, R.F., Fuchs, G., 2010. Fructose 1,6-bisphosphate aldolase/phosphatase may be an ancestral gluconeogenic enzyme. Nature 464, 1077-1081.

Schopf, J.W., Kitajima, K., Spicuzza, M.J., Kudryavtsev, A.B., Valley, J.W., 2018. SIMS analyses of the oldest known assemblage of microfossils document their taxoncorrelated carbon isotope composition. PNAS 115, 53-58.

Shepherd, V.A., 2006. The cytomatrix as a cooperative system of macromolecular and water networks. Curr. Top. Dev. Biol 75, 171-223.

Sleep, N.H., 2010. The hadean-achaean environment. Cold Spring Harb. Perspect. Biol. 2, a002527.

Sleep, N.H., Bird, D.K., Pope, E.C., 2011. Serpentinite and the dawn of life. Phil. Trans. R. Soc. B 366, 2857-2869.
Sousa, F.L., Thiergart, T., Landan, G., Nelson-Sathi, S., Pereira, I.A.C., Allen, J.F., Lane, N., Martin, W.F., 2013. Early bioenergetic evolution. Phil. Trans. R Soc. B 368, 20130088.

Spitzer, J., 2017. 2017. Emergence of life on earth: a physicochemical jigsaw puzzle. J. Mol. Evol. 84, 1-7.

Stüeken, E.E., Anderson, R.E., Bowman, J.S., Brazelton, W.J., Colangelo-Lillis, J., Goldman, A.D., Som, S.M., Baross, J.A., 2013. Did life originate from a global chemical reactor? Geobiology 1, 101-126.

Sutherland, J.D., 2017. Studies on the origin of life- the end of the beginning. Nat Rev. Chem. 1, 0012.

Tashiro, T., Ishida, A., Hori, M., Igisu, M., Koike, M., Méjean, P., Takahata, N., Sano, Y., Komiya, T., 2017. Early trace of life from 3.95 ga sedimentary rocks in labrador, canada. Nature 549, 516-518.

Toxvaerd, S., 2014. The role of carcohydrates at the origin of homochirality in biosystems. Orig. Life. Evol. Biosph. 44, 391-409.

Toxvaerd, S., 2017. The role of the peptides at the origin of life. J. Theor. Biol. 429, 164-169.

Toxvaerd, S., 2018. The start of the abiogenesis: preservation of homochirality in proteins as a necessary and sufficient condition for the establishment of the metabolism. J. Theor. Biol. 451, 117-121.

Velasco, J., 2018. Universal common ancestry, LUCA, and the tree of life: three distinct hypotheses about the evolution of life. Biology \& Philosophy 33, 1-18.

Walker, S.I., 2017. Origins of life: a problem for physics. a key issues review. Rep. Prog. Phys. 80, 092601

Weiss, M.C., Preiner, M., Xavier, J.C., Zimorski, V., Martin, F., 2018. The last universal common ancestor between ancient earth chemistry and the onset of genetics. PLoS GENET 14 (8), e1007518.

Weiss, M.C., Sousa, F.L., Mrnjavac, N., Neukirchen, S., Roettger, M., Nelson-Sathi, S., Martin, W.F., 2016. The physiology and habitat of the last universal common ancestor. Nat. Microbiol. 1, 1-8.

Woese, C.R., 1987. Bacterial evolution. Microbiol. Rev. 51, 221-271.

Woese, C.R., E., F.G., 1977. The concept of cellular evolution. J. Mol. Evol. 10, 1-6. 